\title{
Accretion onto Protoplanetary Discs: Implications for Globular Cluster Evolution
}

\author{
T. P. G. Wijnen ${ }^{1,2}$, O. R. Pols ${ }^{1}$, F. I. Pelupessy ${ }^{2}$ and S. Portegies \\ Zwart $^{2}$ \\ ${ }^{1}$ Department of Astrophysics/IMAPP, Radboud University Nijmegen, \\ P.O. Box 9010, 6500 GL Nijmegen, The Netherlands \\ email: thomas.wijnen@astro.ru.nl \\ ${ }^{2}$ Leiden Observatory, Leiden University, \\ PO Box 9513, 2300 RA Leiden, The Netherlands
}

\begin{abstract}
In the past decade, observational evidence that Globular Clusters (GCs) harbour multiple stellar populations has grown steadily. These observations are hard to reconcile with the classical picture of star formation in GCs, which approximates them as a single generation of stars. Bastian et al. recently suggested an evolutionary scenario in which a second, chemically distinct, population is formed by the accretion of chemically enriched material onto the protoplanetary disc of low-mass stars in the initial GC population. Using assumptions that represent the (dynamical) conditions in a typical GC, we investigate whether a low-mass star surrounded by a protoplanetary disc can accrete sufficient enriched material to account for the observed abundances in 'second generation' stars. We compare the outcome of two different smoothed particle hydrodynamics codes and focus on the lifetime and stability of the disc and on the gas accretion rate onto both the star and the disc.
\end{abstract}

Keywords. accretion, accretion discs, protoplanetary discs, planetary systems: formation, stars: formation, globular clusters: general

\section{Context}

The idea of the early disc accretion scenario is that the massive stars reside in binaries in the cluster core and expel enriched material through binary interactions. The lowmass, pre-main sequence stars of the same generation then sweep up the gas into their protoplanetary disc as they move through the cluster core (Bastian et al. 2013, B13). To explain the most extreme abundance spreads observed in globular clusters, they have to accrete an amount of enriched material of the order of their own mass (as inferred from, e.g., the main sequence of NGC2808, Piotto et al. 2007) within the expected disc lifetime of $10^{6}-10^{7}$ years. We test this scenario by simulating a protoplanetary disc embedded in a laminar flow of gas with 2 smoothed particle hydrodynamics (SPH) codes, Fi (Pelupessy et al. 2004) and Gadget2 (Springel 2005), within the AMUSE framework (Pelupessy et al. 2013). We test against different implementations of the artificial viscosity in each SPH code.

\section{Results \& Implications}

Our comparison shows that the rate at which the protoplanetary disc can sweep up interstellar material (ISM loading rate, the green symbols in Fig. 1a) is consistent for both codes and all resolutions. It is a factor 2 lower than the rate expected from geometrical arguments (and used in B13):

$$
\dot{M}_{\mathrm{ISM}}=\rho_{\mathrm{ISM}} v_{\mathrm{ISM}} \pi R_{\mathrm{disc}}^{2},
$$




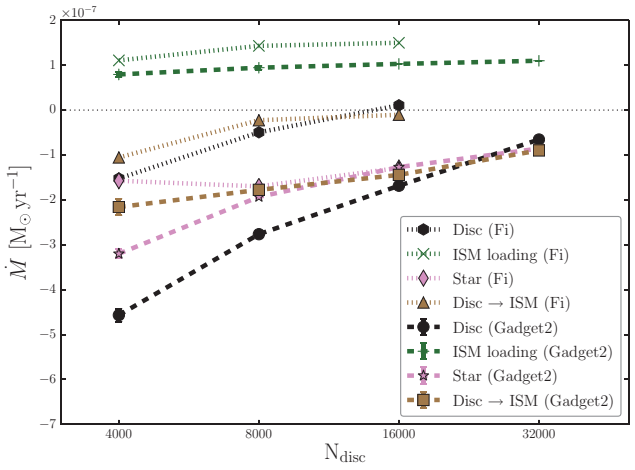

(a) Average mass loss and gain rates as a function of the number of disc particles, $\mathrm{N}_{\mathrm{disc}}$, for both SPH-codes Fi (dotted) and Gadget2 (dashed). The change of the disc mass (black) consists of 3 components: mass is lost due to accretion onto the star (purple) and stripping at the outer edge (brown), and gained from sweeping up ISM by the disc and star (green).

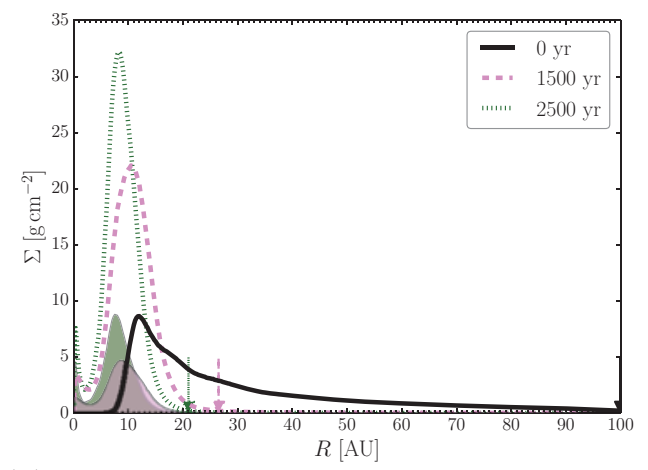

(b) The azimuthally averaged surface density profile for a simulation with Gadget2 and 16.000 disc particles at the start (solid black), after 1500 year (dashed purple) and 2500 year (dotted green). The contribution of sweptup ISM at each moment is plotted in the corresponding transparent colour, while the coloured arrow indicates the disc radius at that moment.

\section{Figure 1.}

where $\rho_{\text {ISM }}$ and $v_{\text {ISM }}$ are the density and velocity of the ISM with respect to the protoplanetary disc and $R_{\text {disc }}$ the radius of the disc. This means that the effective surface area of the disc is smaller than its actual surface area. We can explain this using the surface density profile of the discs at different times (Fig. 1b). The swept up ISM (shaded regions) is only located at smaller radii and never extends to the outer radius of the disc (coloured arrows), indicating that the ISM flows around the disc at the outer edge instead of being entrained by it.

The outer parts of the disc are continuously being stripped (brown symbols in Fig. 1a) by the ram pressure of the flow. The disc therefore not only loses mass (black symbols in Fig. 1a), but also angular momentum. In our simulations, the disc loses both its mass and angular momentum on a time scale of $10^{3}-10^{4}$ years, which would make the early disc accretion scenario infeasible. However, our convergence study suggests that this is a numerical rather than physical effect and that it can be attributed to (the treatment of) the artificial viscosity in the disc. This work will be presented extensively in Wijnen et al. 2015.

\section{References}

Bastian N., Lamers H. J. G. L. M., de Mink S. E., Longmore S. N., Goodwin S. P., \& Gieles M. MNRAS, 436, 2398

Pelupessy F. I., van der Werf P. P., \& Icke V. 2004, A\& $A, 422,55$

Pelupessy F. I., van Elteren A., de Vries N., McMillan S. L. W., Drost N., \& Portegies Zwart S. F. 2013, $A \mathscr{S} A, 557,84$

Piotto G., Bedin L. R., Anderson J., King I. R., Cassisi S., Milone A. P., Villanova S., Pietrinferni A., \& Renzini A. 2007, ApJ, 661, L53

Springel V. 2005, MNRAS, 364, 1105

Wijnen T. P. G.., Pols O. R., Pelupessy F. I., \& Portegies Zwart S. 2015, submitted to MNRAS 